\title{
SECOND ORDER DIFFERENTIAL EQUATIONS WITH RATIONAL COEFFICIENTS
}

\author{
A. HINKKANEN AND JOHN ROSSI
}

(Communicated by Irwin Kra)

\begin{abstract}
The authors exhibit two linearly independent real solutions, $f_{1}$ and $f_{2}$, to $w^{\prime \prime}+H w=0$ such that $f_{1} f_{2}$ is transcendental, $f_{1}$ and $f_{2}$ have only real zeros and poles and $H$ is a nonconstant rational function. This shows the sharpness of a recent result of Hellerstein and Rossi. Some related results are also proved.
\end{abstract}

\section{INTRODUCTION}

This note deals with solutions, meromorphic in the complex plane $\mathbf{C}$, to the differential equation

$$
w^{\prime \prime}+H w=0
$$

where $H$ is a rational function. In general, if $H$ is not a polynomial, (1.1) may admit multivalued solutions. Consequently, throughout this note we assume that $H$ is such that (1.1) admits only solutions single-valued and meromorphic in $\mathbf{C}$.

If $H=P / Q$ where $P, Q$ are polynomials, we denote by $\operatorname{di}(H)$, the degree at infinity of $H$, defined by

$$
\operatorname{di}(H)=\operatorname{deg} P-\operatorname{deg} Q,
$$

where $\operatorname{deg} P$ (respectively $\operatorname{deg} Q$ ) is the degree of $P$ (respectively $Q$ ). We denote by $\operatorname{deg} H$ the usual degree of the rational function $H$, that is, the total number of poles of $H$ in the Riemann sphere, with due count of multiplicity. We also denote by $n_{\mathrm{NR}}(r, f)$ the number of nonreal zeros of a meromorphic function $f$ in a closed disk of radius $r$ centered at the origin.

In a recent paper [2], the following theorem is proved.

Theorem A. Let $H$ be a rational function such that (1.1) admits only meromorphic solutions. Let $f_{1}, f_{2}$ be two linearly independent solutions to (1.1) with

Received by the editors November 13, 1987 and, in revised form, May 26, 1988.

1980 Mathematics Subject Classification (1985 Revision). Primary 34A20; Secondary 30D35.

The first author's research was supported in part by the U. S. National Science Foundation. 
$f_{1} f_{2}$ transcendental. If as $r \rightarrow \infty$

$$
n_{\mathrm{NR}}\left(r, f_{i}\right)=o\left(r^{\frac{1}{2}(\mathrm{di}(H)+2)}\right) \quad(i=1,2)
$$

then

$$
\operatorname{di}(H)=0 .
$$

We remark that (1.1) can never admit only meromorphic solutions if $\operatorname{di}(H)<$ -2 while if $\operatorname{di}(H)=-2$, then $f_{1} f_{2}$ is always rational (c.f. [2, p. 604]). Examples in [2] show that if $H$ is not a polynomial, the transcendentality of $f_{1} f_{2}$ cannot be removed; while if $H$ is a nonconstant polynomial, $f_{1} f_{2}$ is always transcendental (c.f. [1, Theorem 1]).

If we assume $H$ to be a polynomial in Theorem A and if (1.2) holds, then (1.3) implies that $H \equiv c$, where $c$ is a constant and then (1.2) implies that $c>0$. In general, however, the conclusion of Theorem A that $\operatorname{di}(H)=0$ cannot be replaced by $H \equiv c$ since (c.f. [2, p. 604])

$$
f_{1}(z)=e^{i z} z^{-1}(i z-1)
$$

and

$$
f_{2}(z)=\cos z-z^{-1} \sin z
$$

satisfy (1.1) with $H(z)=1-2 z^{-2}$. It is pointed out in [2] that $f_{1} f_{2}$ is transcendental while $f_{1}$ has a single nonreal zero and $f_{2}$ has only real zeros.

A natural question raised by Hellerstein, Williamson, Kohs and the authors asks whether there are any reasonable hypotheses on $f_{1}$ and $f_{2}$ which force $H$ to be constant. The question really asks what properties of sine and cosine $f_{1}$ and $f_{2}$ must possess to force (1.1) to be a sine equation. Thus a reasonable hypothesis might be that $f_{1}$ and $f_{2}$ are real (i.e. $f_{i}(x)$ is real or infinite when $x$ is real) and each has only real zeros and poles. We show that this is not enough.

Theorem 1. There exists a nonconstant real rational function $H$ such that (1.1) has two real, linearly independent solutions $f_{1}$ and $f_{2}$ such that $f_{1} f_{2}$ is transcendental while $f_{1}$ and $f_{2}$ have only real zeros and poles.

Theorem 1 puts very natural restrictions on $f_{1}$ and $f_{2}$. Although further restrictions, say on the zero or pole distribution of $f_{1}$ and $f_{2}$, may force $H$ to be constant, we find this less interesting and do not attempt to study such questions.

We will also investigate properties of $H$ which will allow (1.1) to admit two linearly independent solutions $f_{1}$ and $f_{2}$ with only real zeros. It is easy to construct an example satisfying the conclusions of Theorem 1 if we allow $f_{1} f_{2}$ to be rational. Indeed $f_{1}(z)=e^{z}(z-1) / z$ and $f_{2}(z)=e^{-z}(z+1) / z$ have only real zeros and poles and solve (1.1) with $H(z)=-1-2 z^{-2}$. So as in Theorem A, we assume $f_{1} f_{2}$ to be transcendental which implies that at least one of $f_{1}$ 
and $f_{2}$ has infinitely many zeros [1, Theorem 1]. Then by Theorem $\mathrm{A}$, we have $\operatorname{di}(H)=0$. Also (c.f. [2, Theorem C]), since one solution of $(1.1)$ has infinitely many real zeros and $\operatorname{di}(H)=0$ then $H(\infty)>0$. With no loss of generality we assume that

$$
H(\infty)=1
$$

With this in mind we make the following definition.

Definition. $A$ rational function $H$ is called admissible if it is nonconstant and satisfies (1.6), and if (1.1) admits only meromorphic solutions, at least one of which has infinitely many real zeros.

The statement of Theorem 2 depends on the following characterization of admissible functions, proved in $\S 2$.

Lemma 1. The function $H$ is admissible if and only if there exist real rational functions $R$ and $S$, with $R$ nonconstant, satisfying

$$
R^{\prime}=1+R^{2}-S^{2}
$$

such that the Schwarzian derivative of $F(z)=e^{2 i z}(R(z)+i)(R(z)-i)^{-1}$ satisfies

$$
\{F, z\}=2 H \text {. }
$$

Then there exist solutions $g_{1}$ and $g_{2}$ to (1.1) satisfying

$$
g_{1}(z)=e^{i z} \frac{R(z)+i}{S(z)}
$$

and

$$
g_{2}(z)=e^{-i z} \frac{R(z)-i}{S(z)} .
$$

Let $\mathscr{H}$ (respectively $\mathscr{L}$ ) denote the open upper (respectively lower) halfplane. Theorem 2. Let $H$ be admissible and let $R$ be as in Lemma 1. Then the following holds:

(i) The function $H$ is real.

(ii) If

$$
R(\mathscr{H})=\mathscr{L}
$$

then a solution of (1.1) has only real zeros if and only if it is a constant multiple of

$$
g_{1}+c g_{2}
$$

where $g_{1}$ and $g_{2}$ are as in (1.9) and (1.10) and $|c|=1$. Furthermore each such solution is a constant multiple of a real solution and $H$ has no real poles. 
(iii) If

$$
R(\mathscr{H})=\mathscr{H}
$$

and if (1.1) admits a solution $g$ with only real zeros and infinitely many, then any other such solution is a constant multiple of $g$.

(iv) If neither (1.11) nor (1.12) holds then (1.1) admits at most $d=\operatorname{deg} R$ pairwise linearly independent solutions with only real zeros.

Conversely, suppose that $H$ is admissible with no real poles and that (1.1) has a solution with only real zeros. Then if $g_{1}$ and $g_{2}$ are given by (1.9) and (1.10) and if $|c|=1$, the solution $g_{1}+c g_{2}$ to (1.1) has only real zeros.

Corollary 1. If $H$ is admissible and if (1.1) has at least one solution with only real zeros, then the following three conditions are equivalent:

(a) there are infinitely many pairwise linearly independent solutions to (1.1) with only real zeros;

(b) the function $H$ has no real poles; and

(c) we have $R(\mathscr{H})=\mathscr{L}$ and $R(\mathscr{L})=\mathscr{H}$. Furthermore, in this case there is a one-parameter family of pairwise linearly independent solutions to (1.1) with only real zeros, given by $g_{1}+c g_{2}$, where $|c|=1$ while $g_{1}$ and $g_{2}$ are as in (1.9) and (1.10).

In $\S 5$ examples will be given to show that (ii) is not vacuous and that (iii) is sharp.

\section{Proof of Lemma 1; Lemma 2}

To prove Lemma 1, first assume (1.7) and define $H$ by (1.8). Simple calculations show that $H$ is rational and satisfies (1.6). Furthermore (1.7) implies that $g_{1}$ and $g_{2}$ solve (1.1). Since $R$ is real, the equation

$$
\tan z=R(z)
$$

has infinitely many real solutions and hence $g_{1}+g_{2}$ has infinitely many real zeros. Since $R$ is nonconstant, also $H$ is nonconstant. Hence $H$ is admissible.

Conversely, if $H$ is admissible, we deduce from [4, $\S 15.5$, p. 372] and from (1.6) that there exist two solutions $g_{1}$ and $g_{2}$ to (1.1), with

$$
g_{1}(z)=e^{i z} R_{1}(z)
$$

and

$$
g_{2}(z)=e^{-i z} R_{2}(z)
$$

where $R_{1}$ and $R_{2}$ are rational and not identically zero. Define

$$
R=-i \frac{R_{2}+R_{1}}{R_{2}-R_{1}}
$$


and

$$
S=\frac{R+i}{R_{1}}
$$

Then

$$
S=\frac{R-i}{R_{2}}
$$

and $R$ and $S$ satisfy (1.7). Since

$$
\left\{g_{1} / g_{2}, z\right\}=2 H
$$

(c.f. [3, p. 648]), (1.8) holds. To deduce that $R$ is nonconstant we need to know that $R_{2} / R_{1}$ is nonconstant. If $R_{2} / R_{1}$ is constant then $g_{1} / g_{2}=C e^{2 i z}$ for some constant $C$ so that $2 H=\left\{g_{1} / g_{2}, z\right\}$ is constant, contradicting the fact that $H$ is admissible. Finally, the proof that $R$ and $S$ are real is contained in the following lemma.

Lemma 2. Let $R$ and $S$ satisfy (1.7) and let $g_{1}$ and $g_{2}$ be given by (1.9) and (1.10), and suppose that $R$ is not constant. Then

$$
g_{1}+\lambda g_{2}
$$

has infinitely many real zeros if and only if there exists $\delta \in[0, \pi)$ such that

$$
\tan (z+\delta)=R(z)
$$

has infinitely many real solutions where

$$
e^{-2 i \delta}=\lambda \text {. }
$$

Furthermore, if (2.7) has infinitely many real zeros (or if (2.8) has infinitely many real solutions and (2.9) holds for some $\delta \in[0, \pi)$ ), it follows that $R$ and $S$ are real and that every solution $g_{1}+c g_{2}$, where $|c|=1$, of $(1.1)$ is a constant multiple of a real function with infinitely many real zeros.

Finally $g_{1}+\lambda g_{2}$ has only real zeros if and only if $\tan (z+\delta)=R(z)$ has only real solutions.

Proof. Clearly $z$ is a solution of (2.8) if and only if it is a solution of

$$
e^{2 i z}=-\lambda \frac{R(z)-i}{R(z)+i},
$$

where $\lambda$ and $\delta$ are related by (2.9). Hence, since $R$ and $S$ are rational, if (2.8) has infinitely many real solutions, so does (2.10). Then (1.9), (1.10) and (2.9) imply that (2.7) has infinitely many real zeros.

If (2.7) has infinitely many real zeros then (2.10) has infinitely many real solutions. Then since $\left|e^{2 i z}\right|=1$ for $z \in \mathbf{R}$ and $|(R(\infty)-i) /(R(\infty)+i)|=1$, we must have $|\lambda|=1$. Thus there is $\delta \in[0, \pi)$ satisfying (2.9) and then (2.8) has infinitely many real solutions. (Of course, $\mathbf{R}$ denotes the real axis.)

Next we assume that (2.7) has infinitely many real zeros or, equivalently, that (2.8) has infinitely many real solutions and that (2.9) holds for some $\delta \in[0, \pi)$. 
Then it is clear that $R$ is real since $R$ is rational and (2.8) has infinitely many real solutions. Now by (1.2), $S^{2}$ is real which implies that $S$ is real or purely imaginary. We assume without loss of generality that $R(\infty) \neq \infty$, for otherwise we replace $R$ by $-1 / R$ and $S$ by $S / R$ in (1.7). If $S$ is purely imaginary then (1.7) and the reality of $R$ imply that $R^{\prime}(x) \geq 1$ for $x \in \mathbf{R}$. But since $R(\infty) \neq \infty$, then $R^{\prime}(\infty)=0$ and we have a contradiction. Thus $S$ is real.

Since (2.8) has infinitely many real solutions for all $\delta \in[0, \pi), g_{1}+c g_{2}$ has infinitely many real zeros with $c=e^{-2 i \delta}$. We now show that $g_{1}+c g_{2}$ is a constant multiple of a real function. Since (2.7) has infinitely many real zeros, (2.10) has infinitely many real solutions. Since $|\lambda|=1,(R+i) /(R-i)^{-1}$ maps the real axis into the unit circle. Thus for $|c|=1$, the function

$$
e^{2 i z} \frac{R(z)+i}{c(R(z)-i)}
$$

maps the real axis into the unit circle. Consequently (2.11), (2.5), (2.6), (1.9) and (1.10) imply that

$$
G(z)=\frac{i g_{1}(z)-i c g_{2}(z)}{g_{1}(z)+c g_{2}(z)}
$$

is real. But by Lemma $1,(1.7)$ implies that $g_{1}$ and $g_{2}$ solve (1.1) with $H$ as in (1.8). Hence $G$ is the quotient of two solutions to (1.1) and

$$
G^{\prime}=k /\left(g_{1}+c g_{2}\right)^{2}
$$

where $k$ is the (constant) Wronskian of the two solutions. Since $G$ is real, so is $G^{\prime}$ and hence $g_{1}+c g_{2}$ is a constant multiple of a real function.

It remains to show that (2.7) has only real zeros if and only if (2.8) has only real solutions. Suppose that $(2.8)$ has only real solutions. Then $(2.10)$ has only real solutions. If

$$
\left(g_{1}+\lambda g_{2}\right)\left(z_{0}\right)=0
$$

then either (2.8) holds and $z_{0}$ is real or $z_{0}$ is a pole of $S$ of multiplicity $m$. But then (1.7) implies that $z_{0}$ is a pole of multiplicity $m$ for $R$. Then $z_{0}$ is a solution of (2.10) and is hence real.

Suppose that (2.7) has only real zeros and that

$$
\tan \left(z_{0}+\delta\right)=R\left(z_{0}\right)
$$

Then $z_{0}$ is a solution of $(2.10)$ and hence if $z_{0}$ is a pole of $R$, it must be real. So without loss of generality

$$
R\left(z_{0}\right) \neq \infty .
$$

Also if $S\left(z_{0}\right) \neq 0$, then $z_{0}$ is a zero of (2.7) and is hence real. Thus we assume (2.13), (2.14) and

$$
S\left(z_{0}\right)=0
$$


where $z_{0}$ is a zero of $S$ of multiplicity $m$. Since (2.13) holds, we have $R\left(z_{0}\right) \neq$ $\pm i$ and so, for a suitable branch of $\arctan R$, we have

$$
\arctan R\left(z_{0}\right)=z_{0}+\delta \text {. }
$$

But by (1.7), we have in a neighborhood of $z_{0}$ that $[(\arctan R(z))-z-\delta]^{\prime}=$ $-S^{2} /\left(1+R^{2}\right)$. This, $(2.13)$ and (2.15) imply that $z_{0}$ is a solution of multiplicity $2 m+1$ for (2.8). So $z_{0}$ is a zero of (2.7) and is thus real. This proves Lemma 2. Hence the proof of Lemma 1 is also complete.

\section{Proof of Theorem 1}

Define

$$
R(z)=-1+\frac{a}{z}-\frac{9}{z-c}
$$

and

$$
S(z)=\sqrt{2}\left(1+\frac{21-a}{2 z}-\frac{6}{z-c}\right)
$$

where $a$ is the positive solution to

$$
\sqrt{2 a(a+1)}+a=21
$$

and

$$
c=(5 a-42) / 7 \text {. }
$$

(Note that $a \sim 8.41$ and $c \sim .01$.) Using (3.3) and (3.4) we find that $R$ and $S$ are real and satisfy (1.7). Also since $a<21$ and $c>0$ we find that $S$ has only real zeros. Thus by Lemma $1,(1.9)$ and (1.10), $g_{1}$ and $g_{2}$ solve (1.1) with admissible $H$ as in (1.8). Now if $H$ has a pole at $z_{0}$ then by (1.1) $g_{1}$ has either a zero or a pole at $z_{0}$. In the former case, since $g_{1}$ and $g_{2}$ are linearly independent, $g_{2}$ must have a pole at $z_{0}$ (cf. [1, Lemma 1, (a), (b)]). Thus $H\left(z_{0}\right)=\infty$ implies that either $g_{1}\left(z_{0}\right)=\infty$ or $g_{2}\left(z_{0}\right)=\infty$. So if $g_{1}$ and $g_{2}$ have only real poles then $H$ and every solution $g=\alpha_{1} g_{1}+\alpha_{2} g_{2}$ to (1.1) have only real poles. By Lemma 2 all that remains to be shown is the existence of $\delta_{1}$ and $\delta_{2}$ where $0 \leq \delta_{1}, \delta_{2}<\pi, \delta_{1} \neq \delta_{2}$ such that

$$
\tan \left(z+\delta_{i}\right)=R(z) \quad(i=1,2)
$$

has only real solutions.

To do this, let $h(z)$ denote a branch of $-z+\arctan R(z)$. This is multivalued but well defined in any simply connected domain not containing a $\pm i$-point of $R$. Then

$$
v(z)=\operatorname{Im} h(z)=\frac{1}{2} \log \left|\frac{R(z)+i}{R(z)-i}\right|-\operatorname{Im} z
$$

is well defined in $\mathbf{C}$ and harmonic except at the $\pm i$-points of $R$ where $v= \pm \infty$. We next prove the following auxiliary result where we denote $\{z: v(z)>0\}$ by $\{v>0\}$. 
Lemma 3. There is exactly one component $V$ of $\{v>0\}$ in $\mathscr{H}$. The domain $V$ is bounded, contains an i-point of $R$ and $\partial V \cap \mathbf{R}=\left\{x_{1}, x_{2}\right\}$ where $x_{1}, x_{2}$ are the two distinct zeros of $S$. The function $\operatorname{Re} h(z)$ changes monotonically by $\pi$ as $z$ completely traverses $\partial V$.

Proof. Set $z=r e^{i \sigma}$. Then (3.6) implies that $v(z)=0$ if and only if

$$
\exp (4 r \sin \sigma)=1+4 \frac{\operatorname{Im} R(z)}{|R(z)-i|^{2}} \text {. }
$$

By (3.1) it is clear that for all large $r$, we have

$$
|\operatorname{Im} R(z)| \leq \frac{K|\sin \sigma|}{r},
$$

where $K$ is a constant. Then (3.7), (3.8) and the fact that $|R(z)-i|^{2} \rightarrow 2$ as $z \rightarrow \infty$ imply for $r \geq r_{0}$ that

$$
1+\frac{4|\operatorname{Im} R(z)|}{|R(z)-i|^{2}} \leq 1+\frac{K|\sin \sigma|}{r} \leq 1+4 r|\sin \sigma| \leq \exp (4 r|\sin \sigma|) .
$$

Thus (3.7) holds only if $\sigma=0$ or $\sigma=\pi$ for $r \geq r_{0}$. Hence the only unbounded curve, where $v=0$, is the real axis.

By (1.7)

$$
h^{\prime}(z)=-\frac{S^{2}}{1+R^{2}} .
$$

Since $S$ and $R$ have the same poles, counting multiplicities, $h^{\prime}(z)=0$ only at the two distinct real zeros of $S$, say $x_{1}$ and $x_{2}$. Thus near $x_{1}$ and $x_{2}$, $h(z)-h\left(x_{i}\right)$ behaves like $-A_{i}\left(z-x_{i}\right)^{3}$ where $A_{i}>0$ and $i=1,2$. This means that

$$
\mathbf{R} \subseteq \partial\{v<0\} .
$$

Since $\mathbf{R}$ is the only unbounded curve where $v=0$, every component of $\{v>0\}$ in $\mathscr{H}$ is bounded.

Since $v$ is harmonic except at the $\pm i$-points of $R$, the maximum principle implies that each component of $\{v>0\}$ must contain an $i$-point of $R$. Furthermore since $R(\mathscr{H}) \neq \mathscr{H}, R(\mathscr{H}) \neq \mathscr{L}$ and $R$ is real of degree 2, it is an easy exercise to prove that $R$ has exactly one $i$-point in $\mathscr{H}$. Thus $\mathscr{H} \cap\{v>0\}=V$ is connected. Since $h(z)-h\left(x_{i}\right) \sim-A_{i}\left(z-x_{i}\right)^{3}, A_{i}>0, i=1,2$, near $x_{1}$ and $x_{2}$, there are points arbitrarily close to $x_{1}$ and $x_{2}$ where $v>0$. This and (3.10) imply that

$$
\mathbf{R} \cap \partial V=\left\{x_{1}, x_{2}\right\} .
$$

Since $V$ contains exactly one $i$-point and no $-i$-points of $R$, the argument principle shows that $\operatorname{Re} h(z)$ changes by $\pi$ as $z$ traverses $\partial V$. Suppose that $\partial V=\gamma_{1}+\gamma_{2}$ where $\gamma_{1}$ and $-\gamma_{2}$ are curves joining $x_{1}$ to $x_{2}$. Since $h^{\prime}=0$ only at $x_{1}$ and $x_{2}$ and $v=\operatorname{Im} h=0$ on $\gamma_{1}$ and on $\gamma_{2}, \operatorname{Re} h$ is monotonic 
on $\gamma_{1}$ and $\gamma_{2}$. Since we are traversing the boundary of a single component of $\{v>0\}$ and $h(z)-h\left(x_{2}\right) \sim-A_{2}\left(z-x_{2}\right)^{3}, A_{2}>0$, near $x_{2}$, then had $\operatorname{Re} h$ been increasing on $\gamma_{1}$, it would continue to increase on $\gamma_{2}$. Lemma 3 is proved.

To find $\delta_{1}$ and $\delta_{2}$ for (3.5), set $h\left(x_{1}\right)=\eta_{1}$ and continue $h(z)$ analytically along one of the boundary curves of $V$ to $x_{2}$. Say $h\left(x_{2}\right)=\eta_{2}$. Since $h$ is real on $\partial V, \eta_{1}$ and $\eta_{2}$ are real. Lemma 3 insures that $0 \neq\left|\eta_{1}-\eta_{2}\right|<\pi$. Since $V$ is the only component of $\{v>0\}$ in $\mathscr{H}$ and (3.11) holds, there are exactly two components of $\{v<0\}$, one unbounded, adjacent to $V$ in $\mathscr{H}$. Thus if $z_{0}$ is a nonreal solution of $\tan \left(z+\eta_{j}\right)=R(z)$ where $j=1$ or 2, then $z_{0} \in \partial V$. Also $\operatorname{Re}\left(h\left(z_{0}\right)-\eta_{j}\right)$ must be an integral multiple of $\pi$. But since $h\left(x_{j}\right)=\eta_{j}$ and $\operatorname{Re} h$ increases monotonically by $\pi$ along $\partial V$, we must have $z_{0}=x_{j} \in \mathbf{R}$, a contradiction. We may now take $\delta_{i}=\eta_{i}+n_{i} \pi$ for $i=1,2$ in (3.5), where $n_{1}$ and $n_{2}$ are suitable integers. Theorem 1 is proved.

\section{Proof of Theorem 2}

The proof of (i) follows immediately by Lemma 2 , since $g_{1}+\lambda g_{2},|\lambda|=1$, solves (1.1) and is a constant multiple of a real function.

To prove (ii), suppose that $R(\mathscr{H})=\mathscr{L}$ and note that by Lemma 2 , the equation

$$
\tan (z+\delta)=R(z)
$$

has infinitely many solutions for any choice of $\delta \in[0, \pi)$. Since $\tan (\mathscr{H})=\mathscr{H}$ and $\tan (\mathscr{L})=\mathscr{L}$, all the solutions of $(4.1)$ for $\delta \in[0, \pi)$ are real. Then by Lemmas 1 and 2 we can find $g_{1}$ and $g_{2}$ as in (1.9) and (1.10) such that $g_{1}+c g_{2}$ is a constant multiple of a real function with only real zeros if $|c|=1$. Conversely, if $f$ is a solution of (1.1) with only real zeros then $f=\alpha g_{1}+\beta g_{2}$. If $\alpha \beta \neq 0$ then since $g_{1}$ and $g_{2}$ have finitely many zeros, Picard's theorem shows that $f$ has infinitely many zeros. Thus Lemma 2 gives that $\left|\beta \alpha^{-1}\right|=1$. If $\alpha \beta=0$ then $f$ is a constant multiple of $g_{1}$ or $g_{2}$. But since $R$ is nonconstant and real and $S$ satisfies (1.7), $f$ must have at least one nonreal zero, a contradiction.

To show that $H$ has no real poles, assume that $H\left(x_{0}\right)=\infty$, where $x_{0} \in \mathbf{R}$. Then as we argued in the proof of Theorem $1, x_{0}$ is a pole of either $g_{1}$ or $g_{2}$. By (1.9) and (1.10) either $x_{0}$ is a zero of $S$, or $x_{0}$ is a pole of $R$ which is either not a pole of $S$ or is a pole of $S$ of lower multiplicity. In any case, (1.7) and the reality of $R$ and $S$ imply that $R^{\prime}\left(x_{0}\right) \geq 1$ or, if $R\left(x_{0}\right)=\infty$, that $R^{\prime}(x) \geq 1$ when $x_{0}<x<x_{0}+\varepsilon$, for some positive $\varepsilon$. But since $R(\mathscr{H})=\mathscr{L}$, $R^{\prime}(x)$ is nonpositive for all $x \in \mathbf{R}$, a contradiction. Thus $H$ has no real poles.

The proof of (iv) is similar to that of Theorem 1. By Lemma 1, the function $R$ is real. Furthermore, by replacing $R$ by $-1 / R$, if necessary, we may assume that $R(\infty) \neq \infty$. Thus (3.8) and (3.10) hold for $R$ and every component of $\{v>0\}$ contained in $\mathscr{H}$ is bounded where $v$ is as in (3.6). Also as in the proof 
of (ii), $g_{1}$ and $g_{2}$ have nonreal zeros. Thus if (1.1) admits $M>0$ pairwise linearly independent solutions to (1.1) with only real zeros, the solutions have the form

$$
g_{1}+\lambda_{i} g_{2} \quad(i=1, \ldots, M) .
$$

As before, the functions in (4.2) must have infinitely many zeros and so Lemmas 1 and 2 imply that $\left|\lambda_{i}\right|=1$ for $i=1, \ldots, M$. Also there exist distinct points $\delta_{1}, \ldots, \delta_{M} \in[0, \pi)$ such that

$$
\tan \left(z+\delta_{i}\right)=R(z)
$$

has only real solutions for $1 \leq i \leq M$.

Let $h$ and $v=\operatorname{Im} h$ be as in the proof of Theorem 1. Since $R(\mathscr{H}) \neq \mathscr{L}$, there is at least one $i$-point of $R$ in $\mathscr{H}$ and hence at least one component $V$ of $\{v>0\}$ contained in $\mathscr{H}$.

Let the distinct real zeros of $S^{2} /\left(1+R^{2}\right)$ be $x_{1}, \ldots, x_{k}$ and let $x_{j}$ be a zero of $S^{2} /\left(1+R^{2}\right)$. By (1.7) and the reality of $R$, the multiplicity of the zero at $x_{j}$ is even, say $2 m_{j}$. Also by (1.7), the degree of $S^{2} /\left(1+R^{2}\right)$ is $2 d$ where $d$ is the degree of $R$. Hence $m_{1}+\cdots+m_{k} \leq d$. For $z$ sufficiently close to $x_{j}$, we have

$$
h(z)-h\left(x_{j}\right) \sim-A_{j}\left(z-x_{j}\right)^{m}
$$

where $A_{j}>0$ and $m=2 m_{j}+1$ while $h\left(x_{j}\right)$ is real. Hence the intersection of $\mathscr{H}$ with a small disk centered at $x_{j}$ contains $m_{j}$ domains, which are asymptotically like sectors with vertex at $x_{j}$, where $v>0$. These are separated by $m_{j}+1$ sectors in $\mathscr{H}$ where $v<0$.

If $x_{j} \in \partial V$ and exactly $l$ sectors with vertex at $x_{j}$ are contained in $V$, then, as $z$ traverses $\partial V$ once, $z$ passes through $x_{j}$ also $l$ times, and we may distinguish these times by identifying each of them with an appropriate sector.

Let $V$ contain $\mu$-points of $R$, with due count of multiplicity. By the argument principle, $\operatorname{Re} h(z)$ changes by $\mu \pi$ as $z$ traverses $\partial V$ once. Note that $v=0$ and $h$ is real on $\partial V$. Hence for each $i$ with $1 \leq i \leq M$, the point $z$ passes through $\mu$ points such that $-z-\delta_{i}-\arctan R(z)$ is an integral multiple of $\pi$, that is, through $\mu$ solutions of (4.3). Since all these solutions are real, it follows that $V$ contains $\mu M$ small sectors centered at the points $x_{j}$. But $M \leq \mu M \leq d$ which proves (iv).

The above arguments also show that if $M=d$, then all the zeros of

$$
S^{2} /\left(1+R^{2}\right)
$$

must be real, and the set $\{v>0\}$ must have exactly one component in $\mathscr{H}$, since other components would take up some of the available sectors. Also $\mu=1$ so that $\mathscr{H}$ contains a simple $i$-point of $R$ and no other $i$-points of $R$.

To prove (iii) suppose now, in addition to $R(\mathscr{H}) \neq \mathscr{L}$, that $R(\mathscr{H})=\mathscr{H}$. Note that this remains valid even if we replace $R$ by $-1 / R$. Let $V_{1}, \ldots, V_{p}$ be the components of $\{v>0\}$ in $\mathscr{H}$, and let $V_{j}$ contain $\mu_{j} i$-points of $R$, 
with due count of multiplicity. As above, we see that $V_{j}$ contains $M \mu_{j}$ sectors. Hence

$$
d M=\left(\mu_{1}+\cdots+\mu_{p}\right) M \leq m_{1}+\cdots+m_{k} \leq d
$$

and so $M \leq 1$. This proves (iii), and shows furthermore that if $M=1$, then all the zeros of $S^{2} /\left(1+R^{2}\right)$ must be real.

To prove the last statement of Theorem 2, suppose that $H$ is admissible with no real poles and that (1.1) has a solution with only real zeros. It suffices to show that then $R(\mathscr{H})=\mathscr{L}$, after which the rest follows from (ii) of Theorem 2.

Since $\left\{g_{1} / g_{2}, z\right\}=2 H$, where $g_{1}$ and $g_{2}$ are as in (1.9) and (1.10), the poles of $H$ occur exactly at the points where $g_{1} / g_{2}$ fails to be locally homeomorphic. Since every Möbius transformation and the function $\tan z$ are locally univalent everywhere, since

$$
-i\left[\left(g_{1} / g_{2}\right)+1\right] /\left[\left(g_{1} / g_{2}\right)-1\right]=\tan (z-\arctan R),
$$

and since $(z-\arctan R)^{\prime}=S^{2} /\left(1+R^{2}\right)=T$, say, it follows that those poles of $H$ that are not $\pm i$-points of $R$ (where $\arctan R$ is not defined) occur exactly at the zeros of $T$. Since $R$ is real so that $1+R^{2} \geq 1$ on $\mathbf{R}$, and since $H$ has no real poles, we conclude that $T$ has no real zeros.

Now if $R(\mathscr{H}) \neq \mathscr{L}$, there is an $i$-point of $R$ in $\mathscr{H}$, and hence $\mathscr{H}$ contains a component $V$ of $\{v>0\}$ where $v$ is as in (3.6). Arguing as before, we see that since (1.1) has a solution with only real zeros, the set $\mathbf{R} \cap \partial V$ is not empty, and furthermore, $\mathbf{R} \cap \partial V$ consists of finitely many points, each of which is a zero of $T$. Since $T$ has no real zeros, this is a contradiction. Thus $R(\mathscr{H})=\mathscr{L}$, and Theorem 2 is proved.

Corollary 1 is a trivial consequence of Theorem 2 .

We remark in passing that by (4.4), we have

$$
2 H=\{\exp [2 i(z-\arctan R)], z\}=2 T^{2}+\left(T^{\prime} / T\right)^{\prime}-\frac{1}{2}\left(T^{\prime} / T\right)^{2} .
$$

One can also verify that

$$
H=1-2 R^{\prime}+2 R S^{\prime} / S+\left(S^{\prime} / S\right)^{\prime}-\left(S^{\prime} / S\right)^{2} .
$$

which implies that $H$ cannot have additional poles at the simple zeros of $1+R^{2}$ as by (1.7), $S$ remains finite and nonzero at such points. However, $H$ will have a pole at each multiple zero of $1+R^{2}$, if any, and such points are poles of $T$.

\section{EXAMPLES}

(a) To show that part (ii) of Theorem 2 is not vacuous we only need to find $R, S$ real rational satisfying (1.7) with $R(\mathscr{H})=\mathscr{L}$. Define

$$
R(z)=a\left(\frac{1}{z}+\frac{1}{z-1}\right)
$$

where $a$ is the unique positive solution to

$$
\sqrt{a}(1+2 a)=\sqrt{1+a} .
$$


Clearly $R(\mathscr{H})=\mathscr{L}$ and a calculation shows that $S$ satisfies (1.7) where

$$
S(z)=-1+\sqrt{a(a+1)}\left(\frac{1}{z}-\frac{1}{z-1}\right) .
$$

For completeness note that $R$ and $S$ give rise to solutions $g_{1}$ and $g_{2}$ of (1.1) where

$$
\begin{aligned}
& g_{1}(z)=e^{i z} \frac{a(2 z-1)+i z(z-1)}{-z(z-1)-a(1+2 a)}, \\
& g_{2}(z)=e^{-i z} \frac{a(2 z-1)-i z(z-1)}{-z(z-1)-a(1+2 a)},
\end{aligned}
$$

and

$$
H(z)=1-\frac{2(2 z-1)^{2}}{[z(z-1)+a(1+2 a)]^{2}}+\frac{4(2+a)}{z(z-1)+a(1+2 a)} .
$$

(b) Theorem 2, part (iii) is sharp. Indeed $f_{2}$ in (1.5) is real with only real zeros. The associated $R$ for $w^{\prime \prime}+\left(1-2 z^{-2}\right) w=0$ is $R(z)=z$ which maps $\mathscr{H}$ onto $\mathscr{H}$.

(c) In Theorem 2, part (iv), the number $d$ is most likely sharp. One needs to find $R$ satisfying (1.7) with one $i$-point of $R$ and $d-1-i$-points of $R$ in $\mathscr{H}$ such that the boundary of the unique, bounded component $V$ of $\{v>0\}$ in $\mathscr{H}$ intersects $\mathbf{R}$ at $d$ distinct points. Theorem 1 shows that the bound $d$ is sharp if $d=2$.

\section{REFERENCES}

1. S. Bank and I. Laine, On the zeros of meromorphic solutions of second-order linear differential equations, Comment Math. Helv. 58 (1983), 656-677.

2. S. Hellerstein and J. Rossi, Zeros of meromorphic solutions of second order linear differential equations, Math. Z. 192 (1986), 603-612.

3. E. Hille, Lectures on ordinary differential equations, Addison-Wesley, Reading, Mass., 1969.

4. E. Ince, Ordinary differential equations, Dover, New York, 1956.

Department of Mathematics, University of Michigan, Ann Arbor, Michigan 48109

Department of Mathematics, Virginia Polytechnic Institute and State University, BLACKSBURG, VIRGINIA 24061

Current address (A. Hinkkanen): Department of Mathematics, University of Texas at Austin, Austin, Texas 78712 